\title{
Multi-Sensor Wearable Health Device Framework for Real-Time Monitoring of Elderly Patients Using a Mobile Application and High-Resolution Parameter Estimation
}

\section{OPEN ACCESS}

Edited by:

Elisa Pedroli,

Italian Auxological Institute (IRCCS),

Italy

Reviewed by:

Esteban Armas Vega,

Complutense University of Madrid,

Spain

Cosimo Tuena

Italian Auxological Institute (IRCCS),

Italy

*Correspondence:

Gabriel P. M. Pinheiro

gabrielpmp@ieee.org

Specialty section:

This article was submitted to

Brain Health and Clinical

Neuroscience,

a section of the journal

Frontiers in Human Neuroscience

Received: 30 July 2021 Accepted: 22 December 2021

Published: 17 January 2022

Citation:

Pinheiro GPM, Miranda RK,

Praciano BJG, Santos GA,

Mendonça FLL, Javidi E, da

Costa JPJ and de Sousa RT Jr (2022) Multi-Sensor Wearable Health Device Framework for Real-Time Monitoring of Elderly Patients Using a Mobile Application and High-Resolution

Parameter Estimation

Front. Hum. Neurosci. 15:750591. doi: 10.3389/fnhum.2021.750591
Gabriel P. M. Pinheiro ${ }^{1 *}$, Ricardo K. Miranda ${ }^{1}$, Bruno J. G. Praciano ${ }^{1}$, Giovanni A. Santos ${ }^{2}$, Fábio L. L. Mendonça ${ }^{2}$, Elnaz Javidi ${ }^{1}$, João Paulo Javidi da Costa ${ }^{1,2,3}$ and

Rafael T. de Sousa $\mathrm{Jr}^{2}$

${ }^{1}$ Department of Mechanical Engineering, University of Brasilia, Brasilia, Brazil, ${ }^{2}$ Department of Electrical Engineering, University of Brasilia, Brasilia, Brazil, ${ }^{3}$ Department 2-Campus Lippstadt, Hamm-Lippstadt University of Applied Sciences, Hamm, Germany

Automatized scalable healthcare support solutions allow real-time $24 / 7$ health monitoring of patients, prioritizing medical treatment according to health conditions, reducing medical appointments in clinics and hospitals, and enabling easy exchange of information among healthcare professionals. With recent health safety guidelines due to the COVID-19 pandemic, protecting the elderly has become imperative. However, state-of-the-art health wearable device platforms present limitations in hardware, parameter estimation algorithms, and software architecture. This paper proposes a complete framework for health systems composed of multi-sensor wearable health devices (MWHD), high-resolution parameter estimation, and real-time monitoring applications. The framework is appropriate for real-time monitoring of elderly patients' health without physical contact with healthcare professionals, maintaining safety standards. The hardware includes sensors for monitoring steps, pulse oximetry, heart rate $(\mathrm{HR})$, and temperature using low-power wireless communication. In terms of parameter estimation, the embedded circuit uses high-resolution signal processing algorithms that result in an improved measure of the HR. The proposed high-resolution signal processing-based approach outperforms state-of-the-art HR estimation measurements using the photoplethysmography (PPG) sensor.

Keywords: embedded high-resolution parameter estimation, healthcare multi-sensor wearable hardware development, health monitoring application architecture, ESPRIT, photoplethysmography

\section{INTRODUCTION}

Nowadays, health systems, including hospitals and their intensive care units (ICU), are challenged by a substantial need to increase critical care capacity due to the Coronavirus Disease 2019 (COVID-19) pandemic (Phua et al., 2020). This same paper highlights the importance of streamlining workflows for rapid diagnosis and isolation, clinical management, and infection prevention essential to caring for COVID-19 patients. It also emphasizes the need to protect healthcare workers and other patients while supporting ICU practitioners' activities, hospital administrators, governments, and policymakers. As elderly people are more vulnerable to 
COVID-19, according to Rezende et al. (2020), and other diseases, it is important to ensure continuing healthcare to this part of the population using no-contact methods.

As suggested in Alwashmi (2020), health systems invest in automatized and scalable digital health support solutions, such as healthcare wearable devices and information systems empowered with artificial intelligence. Such automatized digital health solutions allow real-time $24 / 7$ health monitoring of patients, prioritizing medical treatment according to the patients' health conditions, reducing medical appointments in clinics and hospitals, by sharing secure information among healthcare professionals. This is especially of interest when treating the elderly, as they are at greater risk in hospital environments as concluded in Costantino et al. (2021). Therefore, the framework presented in this paper can enable fewer hospital admissions of elderly people while closely monitoring their health and prioritizing more severe cases.

According to Grand View Research (2020), the global market for wearable medical devices was valued at USD 13 billion in 2019, with an expected annual growth rate of $27.9 \%$ until 2027. Still, according to Grand View Research (2020), multisensor health wearable devices are becoming popular due to the cost reduction of remote health monitoring technologies, including home healthcare. Moreover, an affordable and precise health-centered wearable device can allow for novel monitoring infrastructure and increase patients' quality of life.

In this paper, we propose a multi-sensor wearable health device (MWHD) framework with a real-time monitoring application and high-resolution parameter estimation. The proposed hardware includes sensors for step counting, pulse oximetry, heart rate (HR), and temperature measurements. Since wireless communications require a significant consumption of device battery energy (de Freitas et al., 2012; Marinho et al., 2013), the proposed MWHD optimizes the battery usage by using Bluetooth Low Energy (BLE). In terms of parameter estimation, the embedded integrated circuit programmed with high-resolution signal processing algorithms processes the sensors' signals, allowing improved analysis of the steps, pulse oximetry, and HR. Finally, the patient's medical information is reliably provided by the real-time monitoring application to healthcare workers.

This paper is composed of five sections, including this introduction as section 1. Section 2 shows related works in health data signal processing and healthcare platforms. Section 3 proposes a wearable device prototype using photoplethysmography (PPG), an algorithm for HR estimation, and a software platform architecture for remote healthcare supervision. Section 4 presents the methodology and results of the performance comparison of PPG processing algorithms for HR estimation. Finally, section 5 concludes the paper.

\section{STATE OF THE ART}

In Wu et al. (2020), the feasibility of a compact wearable sensor patch for measurements of different physiological signals, including PPG and body temperature, is presented. The wearable sensor system transmits the physiological measurements wirelessly to a gateway using a BLE module. The health data is encrypted, stored, and analyzed on the Internet cloud.

Concerning the usefulness and acceptance of wearable devices among the elderly population, paper (Kekade et al., 2018) presents a systematic review and survey results. The authors show that fewer elderly people are using wearable devices, while more than $60 \%$ of them were interested in using such devices. This presents an opportunity to expand the adoption of wearables in elderly healthcare. Also, only $26 \%$ percent of individuals were willing to pay for a wearable device, showing a need to ally their use with healthcare systems and not necessarily personal use. Finally, despite the disadvantages of wearables, the paper found good compliance prospects and prescribed raising awareness of the technology.

In Puranik and Morales (2020), a digital filter for PPG signals collected from an MWHD is proposed using an adaptive neural network, allowing a more accurate estimate of the HR, resulting in a variation of $3 \%$ concerning the ground truth. In Chung et al. (2020), a deep learning approach is proposed for the HR estimation using PPG signals, achieving an absolute error of 1.5 beats-per-minute (BPM), outperforming state-of-the-art methods. Finally, the authors of Panwar et al. (2020) present a new deep learning model with the capability to estimate HR using only a single channel provided by the PPG signal, achieving an error for HR estimation of 0.046 BPM. Note that the usage of neural networks requires labeled data and the tuning of the neural network hyperparameters.

In Coffen et al. (2020), a new ring-shaped sensor is proposed to estimate the heartbeat using reflective PPG. The measurements are transmitted to a mobile phone via Bluetooth 4.0. Compared with the commercial solution, the ring-shaped sensor presented an error of $2 \%$ smaller.

Still, regarding form-factor preference and acceptance, authors of Kolasinska et al. (2018) assess the usability of a set of sensors hidden in everyday objects. When prompted which pieces of jewelry were more frequently used, seniors gave the two most common answers: a watch $(39 \%)$ or a bracelet $(39 \%)$. Besides, health practitioners found greater functionality in writslocated devices. The paper also found other indications of the willingness of elderly people to use such wearable devices.

In Wang et al. (2019), it improves the HR estimation by using a new notch filter, and the noise cancellation approach is based on the least mean squares. As a result, the error of the HR estimation employing this approach is smaller than 1 BPM using measurements from intensive physical activities.

Reference (Xiong et al., 2017) discusses challenges in HR estimation from a wrist band with PPG collected during intense exercises. First, Principal Component Analysis (PCA) and adaptive filtering are used for removing noise from the PPG signals. Then, to estimate the HR, a Support Vectors Machine (SVM) based approach is considered. The approach in Xiong et al. (2017) presents errors of 1.01 BPM.

In Przybyło (2019), a new method is proposed to estimate HR using PPG. The method also includes Blind Source Separation (BSS) to improve the results further. The achieved Root-MeanSquare Error (RMSE) is 6.1 BPM. 
In Godfrey (2017), authors discuss limitations shown in commercial wearable devices, citing the main limitations as nonclinically oriented devices and failure to provide transparent functionality on account of proprietary software. The authors propose that wearable devices can accommodate the needs of older adults while simultaneously monitoring gait due to their importance in checking aging-related pathologies. The paper (Godfrey, 2017) concludes by recommending the use of multiple and higher-resolution processing algorithms to overcome current limitations in gait assessment.

A novel health wearable platform for the real-time monitoring of accidents of elderly people is shown in Lampoltshammer et al. (2014). In terms of performance, the improved sensors in Lampoltshammer et al. (2014) present a longer battery lifetime, allowing their usage by elderly people for long periods.

Authors present another similar system in Durán-Vega et al. (2019). The presented framework is used in real-time monitoring of patients of nursing homes and comprises a wearable device, mobile application, and necessary middleware. The paper presents assessments for more convenient use of both the wearable and the application on the patients and of their caregivers. Our solution diverges by focusing on any clinical setting, employing a high-resolution processing algorithm aiming for clinical-appropriate data. Besides, our solution also looks to lower the cost of the device, even more, keeping it around \$25 US.

\subsection{State-of-The-Art Simplified Model for PPG Waveforms}

The PPG waveform can be modeled as a pulsating quasiperiodic component attributed to synchronous cardiac changes in the blood volume with each heartbeat. A slowly varying low-frequency component superimposes this pulsating component, with various lower frequency components attributed to respiration, sympathetic nervous system activity, and thermoregulation (Allen, 2007). The PPG signal is sampled with a sampling rate of $f_{s}$. Such sampled PPG waveform is modeled by:

$$
x[n]=A \cos [2 \pi f n+\theta]+r[n]
$$

where $A$ is the PPG signal amplitude, $f$ is the relative frequency of the PPG signal normalized by $f_{s}, \theta$ is the phase of the PPG signal, and $r[n]$ is a component that comprises the noise and artifacts present in the PPG signal. Note that, due to the HR variability (HRV) (Buccelletti et al., 2009) and the Inter-Beat Intervals (IBI), $\mathrm{HR}$ is a time variable. However, we assume a short estimation interval, such that the model in (1) can be applied.

HR detection can then be formulated as a frequency estimation problem. Thus, by measuring the frequency parameter $f$ of a periodic heart signal, given in $\mathrm{Hz}$, we convert the HR to the corresponding value in BPM, given by $\mathrm{BPM}=f \cdot 60$.

In the literature, common approaches for the HR estimation that are applied in embedded systems include Fast-Fourier Transform (FFT) based (Santamaria et al., 2000), autocorrelation (Proakis, 2013), zero-crossing detection (Zhang et al., 2008) and peak detection (Scholkmann et al., 2012). These approaches are summarized in Appendices A.1, A.2, A.3, and A.4, respectively.

\section{PROPOSED HEALTHCARE PLATFORM COMPOSED OF LOW-COST HARDWARE, HIGH-RESOLUTION PARAMETER ESTIMATION ALGORITHMS, AND REAL-TIME MONITORING APPLICATION ARCHITECTURE}

This section, as depicted in Figure 1 proposes the multisensor health wearable device framework with real-time monitoring application and high-resolution parameter estimation. According to Figure 1, each patient is assigned an MWHD, which gathers and processes each subject's health data. Patients can be isolated from each other and healthcare professionals, reducing contact to only the necessary to enforce health protocols. This approach better fits the needs for tighter healthcare procedures with seniors. The MWHD can transmit the processed data via a wireless communication protocol based on BLE to a concentrator device. Note that the concentrator hardware depicted in Figure 1 has been proposed in Prettz et al. (2017). This concentrator device uploads data to a cloud server, which interacts with the proposed monitoring application installed on mobile healthcare workers. The healthcare workers are thus able to monitor patients and collaborate in real-time.

This section is divided into three subsections. In section 3.1, we detail the proposed MWHD. In section 3.2, we propose the high-resolution processing algorithm for $\mathrm{HR}$ estimation. In section 3.3, we propose the healthcare platform for realtime monitoring.

\subsection{Proposed Healthcare Wearable Device Including Multiple Sensors, Processor, and Low Energy Wireless Communication}

The proposed MWHD prototype shown in Figure 2 is batteryoperated and contains sensors to measure the health information of the user-namely HR, pulse oximetry, body temperature, and steps. The current paper focuses on high-resolution HR measurements. Additionally, an application of step counting is shown in Rega et al. (2019). We also implemented the other parameters as directed by the manufacturer's datasheet of the MAX30102 pulse oximetry sensor from Maxim Integrated (2018). The sensors communicate over an Inter-Integrated Circuit $\left(\mathrm{I}^{2} \mathrm{C}\right)$ bus with the microcontroller unit (MCU). The MCU then transmits data to mobile devices using BLE, thus minimizing power consumption.

Selected Area A of Figure 2 comprises the CC2640R2F Cortex $^{\circledR}-\mathrm{M} 3$ MCU circuit by Texas Instruments (2016) and additional components for operating the MCU and for the BLE communication interface. Two crystals are positioned to generate clock signals for different modes of the MCU, one with $24 \mathrm{MHz}$ for regular speed operation and another with $32 \mathrm{kHz}$ for lowpower mode. The reduced speed during low-power mode saves power during idle operation. The General Purpose Input/Output 


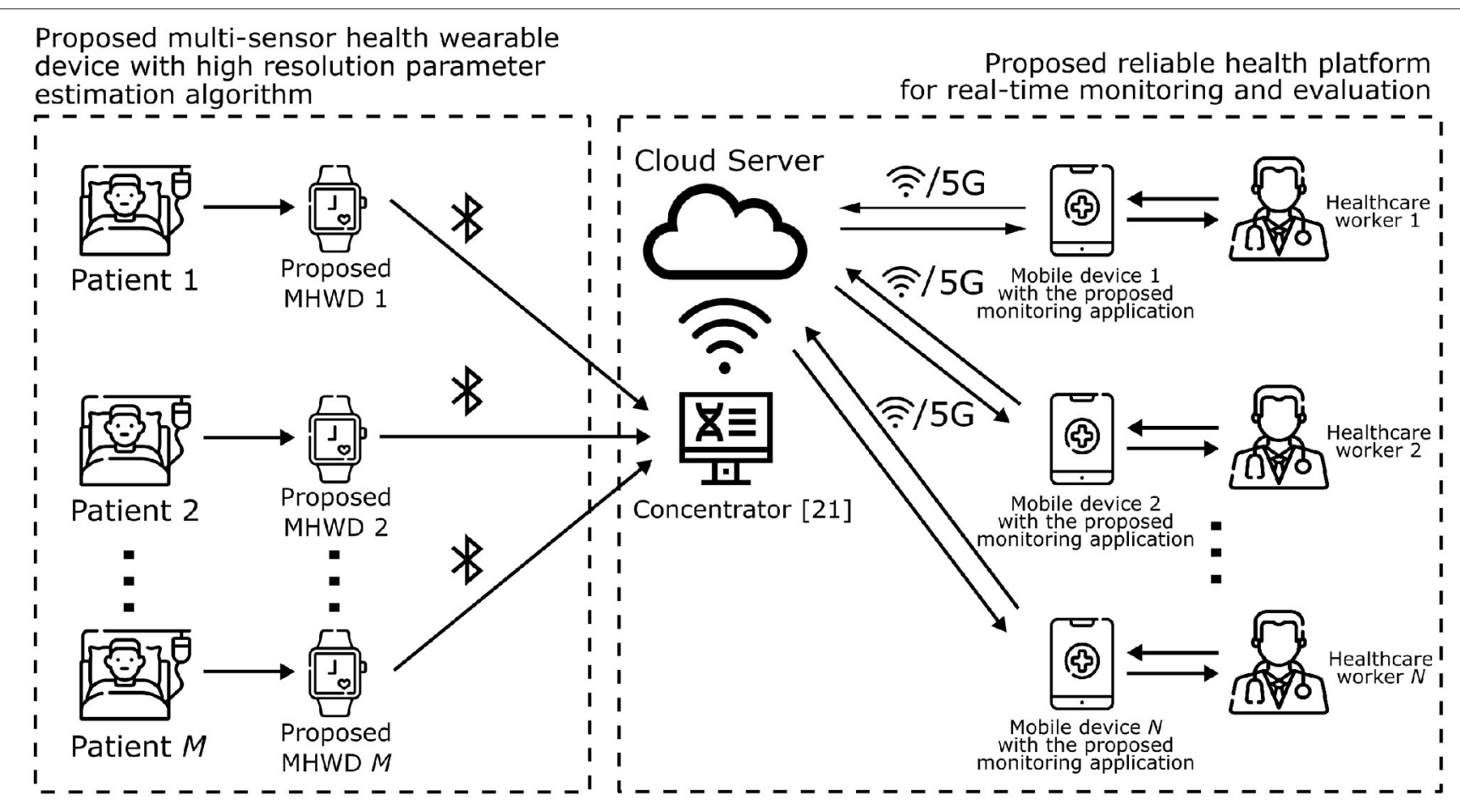

FIGURE 1 | Diagram of the proposed healthcare framework, consisting of a multi-sensor health wearable device, PPG high resolution parameter estimation, and real-time monitoring application.

(GPIO) pins and Joint Test Action Group (JTAG) Interface are made available in pin connectors for ease of access during testing of the MWHD prototype. The Radiofrequency (RF) circuitry is built according to the recommendations by Texas Instruments (2016) related to the $4 \mathrm{x} 4$ External Single-ended configuration, which requires a smaller board space and saves more power. Two push buttons are added for user input and interaction with the device.

Selected Area B of Figure 2 is the power sourcing part of the circuit from a small Lithium-Polymer (LiPo) battery. The nominal $3.7 \mathrm{~V}$ of the LiPo battery is regulated to 3.3 and $1.8 \mathrm{~V}$ using low-dropout regulators LP5907MFX-3.3 and LP5907MFX1.8. The regulators guarantee a stable voltage of operation for the MCU and sensors during device operation and battery's charge and discharge cycles. In addition, the LiPo battery is equipped with a generic commercial micro USB recharging module with overcharge, over-discharge, and current protections.

Selected Area C of Figure 2 includes the sensors for health data measurements. The signals provided by the PPG sensor MAX30102 are used to estimate the HR and pulse oxymetry using red or infrared (IR) LEDs. In conjunction, red and IR LED signals enable pulse oximetry estimation based on the different absorption rates of arterial and venous blood. The prototype device also comprises an accelerometer LIS331DLTR, used for the step counter, to measure movement artifacts used for interference reduction on the PPG signal. For keeping track of body temperature variations, such as fever, the prototype utilizes a MAX30205 sensor. The sensor is positioned on the bottom side of the prototype device to contact the users' skin. The values read are calibrated according to the manufacturer's specifications in Maxim Integrated (2016).

The proposed printed circuit board (PCB) is designed as a 4-layer board to achieve routing requirements and reduce electromagnetic interference (EMI). Additionally, the proposed PCB is double-sided mounted, with a central processor and input buttons on the top and sensors on the bottom to contact the user's wrist skin (required by PPG and body temperature measurements). Test points are also positioned for power sourcing, communication interfaces, and JTAG debugging.

Figure 3 shows photos of the MWHD prototype produced according to the block diagram of Figure 2. On the left-hand side photo, the complete prototype encapsulated as a smartband is shown, while on the right-hand side photo, the PCB of the block diagram of Figure 2 is depicted.

The MWHD is projected as a low-cost device, enabling a cheaper complete healthcare solution to be deployed on a large scale. The cost of the components and PCB was USD 25.00 in a low volume run, indicating that bulk production can make further cost reductions. The final price of the device is onpar with other low-cost fitness wearable devices available in the market. This cost compares well with clinical health devices, which can cost more than USD 100.00 by a considerable margin.

We carried out the hardware prototype development and the proposed algorithm for HR estimation simultaneously. In addition, we gathered HR data from a MAX30100 device for experimental validation. This different PPG sensor module is 


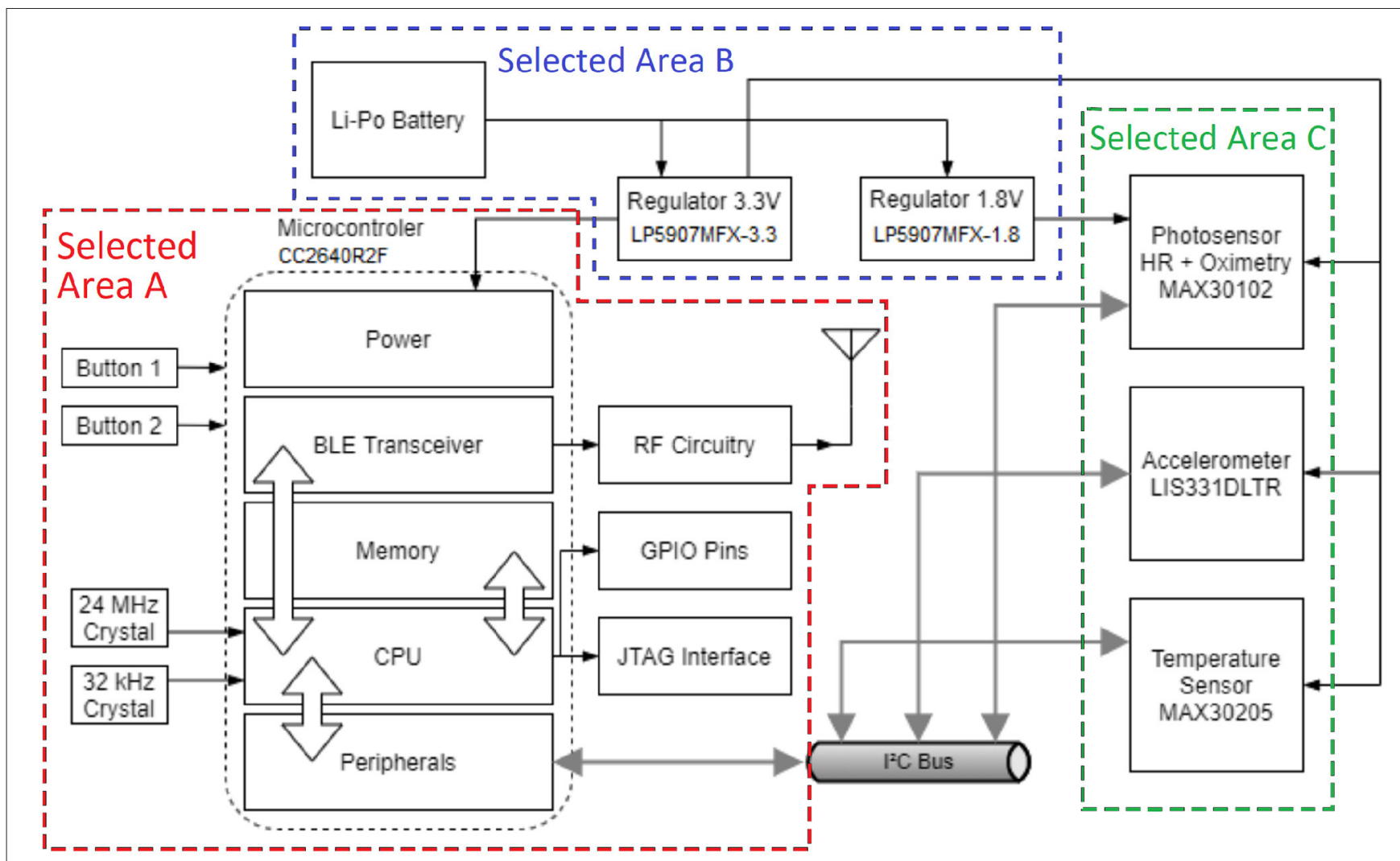

FIGURE 2 | Block diagram showing the functional circuit components of the proposed prototype wristband.

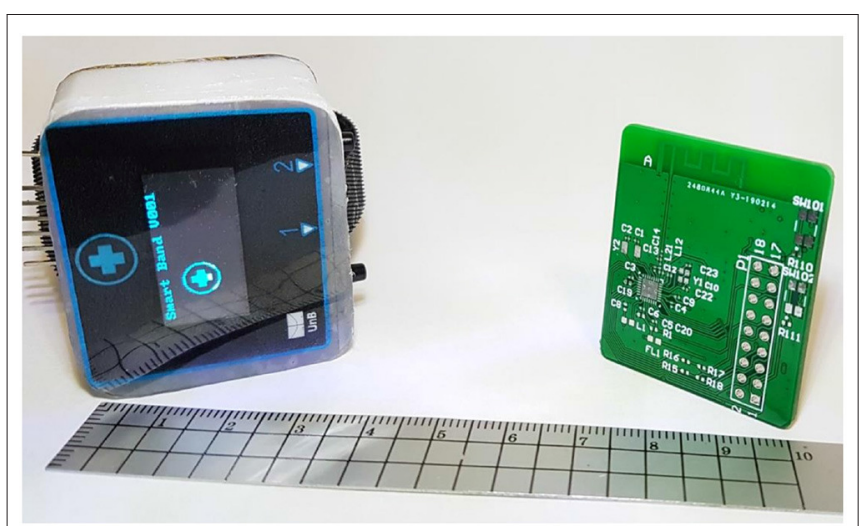

FIGURE 3 | Dimensions of the PCB and enclosure produced for the proposed MWHD device prototype.

equivalent to the projected prototype. The employed pulse oximeter and HR sensor integrated circuit captured data in peripheral oxygen saturation $\left(\mathrm{SPO}_{2}\right)$ measuring mode, though pulse oximetry data is not presently considered. Furthermore, data from the IR LED from the sensor is considered during the tests due to reduced interference from ambient background lighting, while the red LED data is disregarded for the application.
The MAX30100 PPG sensor module communicates via $\mathrm{I}^{2} \mathrm{C}$ bus to the main MCU, which then transmits gathered data to the computer's serial port via a USB-to-Serial adapter. The experiment uses wired serial transmission to guarantee signal integrity further, avoiding adverse effects to any samples input to the algorithms due to events such as package drop or wireless interference.

\subsection{Proposed High Resolution Signal Processing Algorithm for HR Estimation}

A novel approach for HR detection applying the Estimation of Signal Parameters by Rotational Invariance Techniques (ESPRIT) (Roy and Kailath, 1989) algorithm is presented in this subsection. In Reis et al. (2017), the high-resolution signal processing technique named SPHINS is successfully applied for the frequency estimation in forensic applications. In Rega et al. (2019), a high accuracy step counter algorithm based on ESPRIT has been proposed using the accelerometer signals acquired by the sensors of our MWHD prototype proposed in section 3.1. Inspired by the outstanding results of Reis et al. (2017), Reis et al. (2016), and Rega et al. (2019), we propose the usage of the high-resolution signal processing algorithm ESPRIT to measure the HR.

The algorithm exploits the property of rotational invariance of signal subspaces spanned by two temporally displaced data sets 
(Roy and Kailath, 1989). A simplified description of the leastsquared version of the ESPRIT algorithm is shown next based on Manolakis et al. (2005) and Reis et al. (2016).

By applying the Hilbert transform on the PPG waveform model in (1), we obtain the analytic representation of the signal $x[n] \in \mathbb{R}$ given by:

$$
y[n]=x[n]+j \mathcal{H}\{x[n]\},
$$

where the operator $\mathcal{H}\{\}$ denotes the Discrete Hilbert Transform (DHT) and $j=\sqrt{-1}$.

We replace (1) into (2) and rewrite $y[n] \in \mathbb{C}$ by taking into account that $\mathcal{H}\{\}$ is a linear operator, as:

$y[n]=A \cos [2 \pi f n+\theta]+j \mathcal{H}\{A \cos [2 \pi f n+\theta]\}+r[n]+j \mathcal{H}\{r[n]\}$.

The signal $y[n]$ can then be represented as a sum of the complex exponentials with added noise component $w[n]$, comprising the real and imaginary parts of the noise component $r[n]$ :

$$
y[n]=A \exp [2 \pi f n+\theta]+w[n] .
$$

By segmenting the samples obtained by $y[n]$ in (4), we can build a data matrix $\mathbf{Y}$ where $N$ is the amount of data records of the length- $P$ time-window vector signal $y[n]$, thus:

$$
\mathbf{Y}=\left[\begin{array}{llll}
\mathbf{y}[0] & \mathbf{y}[1] & \cdots & \mathbf{y}[N-1]
\end{array}\right]^{\mathrm{T}} \in \mathbb{C}^{P \times N}
$$

where $\mathbf{y}[n]=[\hat{y}[n] \hat{y}[n+1] \ldots \hat{y}[n+P-1]]^{\mathrm{T}}$, and ${ }^{\mathrm{T}}$ is the transposition operator of matrices.

Next we compute the sample covariance matrix of (5) as follows:

$$
\mathbf{R}_{y}=\frac{\mathbf{Y Y}^{\mathrm{H}}}{N} .
$$

By applying the Eigenvalue Decomposition (EVD) in (6), we obtain the following expression:

$$
\mathbf{R}_{y}=\mathbf{U} \Sigma \mathbf{U}^{\mathrm{H}},
$$

where $\mathbf{U}$ is an $P \times P$ matrix of right singular vectors and ${ }^{\mathrm{H}}$ denotes the Hermitian operator. Matrix $\Sigma \in \mathbb{R}^{N \times P}$ has dimensions $N \times P$ and is composed of singular values.

Matrix $\mathbf{U}$ can be decomposed as $\mathbf{U}=\left[\mathbf{u}_{y_{0}} \mid \mathbf{U}_{\mathbf{w}}\right]$, where $\mathbf{u}_{y_{0}}$, the first column of $\mathbf{U}$, is the vector that generates the signal subspace, of dimensions $P \times 1$, formed by the singular vector corresponding to the maximum singular value of the data matrix Y. The remaining singular vectors form a matrix in which its columns correspond to the basis that generate the noise subspace $\mathbf{U}_{\mathrm{w}}$, of dimensions $P \times(P-1)$, orthogonal to the signal subspace.

By writing vectors $\mathbf{u}_{u}$ and $\mathbf{u}_{d}$ formed by the first and last $P-1$ elements of $\mathbf{u}_{y_{0}}$, respectively, the rotational invariance presented previously, and exploited by ESPRIT, guarantees that:

$$
\mathbf{u}_{u} \phi=\mathbf{u}_{d}
$$

where $\phi \in \mathbb{C}$ corresponds to the rotation scalar. By solving (8), the phase value estimation of $\phi$ is given by:

$$
\phi=L \frac{\mathbf{u}_{u}^{\mathrm{H}} \mathbf{u}_{d}}{\mathbf{u}_{u}^{\mathrm{H}} \mathbf{u}_{u}},
$$

where $L$ is the phase notation that denotes the phase angle value of the corresponding complex number.

We determine the frequency estimator $\hat{f}$ with the computed phase angle value of $\phi$ and the sampling frequency $f_{s}$, thus:

$$
\hat{f}=\frac{\phi}{2 \pi} \cdot f_{s}
$$

To estimate HR, we calculate the time window $T$ of the measurement given by $T=n_{\text {samples }} / f_{s}$, that is equivalent to the duration of the measurement in seconds. Finally, the estimated value is $\mathrm{BPM}=\hat{f} \cdot 60 / T$.

Next, we present the summarized steps of the ESPRIT based algorithm for the HR estimation.

$\overline{\text { Algorithm 1: Proposed ESPRIT-based HR estimation via Hilbert }}$ Transform

Given signal $x[n]$ in (1), sampled with frequency $f_{s}$, during a time window of $T$ :

1) Obtain signal $y[n]$ according to (3) by applying the Discrete Hilbert Transform to $x[n]$.

2) Segment the samples obtained in signal $y[n]$ to obtain the data matrix $\mathbf{Y}$ in (5)

3) Compute the sample covariance matrix estimate $\mathbf{R}_{\mathbf{y}}$ of the data matrix $\mathbf{Y}$ as in (6).

4) Decompose $\mathbf{R}_{\mathbf{y}}$ in the corresponding EVD matrices to calculate by eigendecomposition matrix $\mathbf{U}$ according to (7), whose columns are the corresponding right eigenvectors of $\mathbf{R}_{\mathbf{y}}$.

5) Determine the column $\mathbf{u}_{y_{0}}$ of matrix $\mathbf{U}$, corresponding to the maximum singular value of data matrix $\mathbf{Y}$.

6) Determine $\mathbf{u}_{u}$ and $\mathbf{u}_{d}$ by taking the first and last $P-1$ elements of vector $\mathbf{u}_{y_{0}}$.

7) Estimate the rotation scalar $\phi \in \mathbb{C}$ from vectors $\mathbf{u}_{u}$ and $\mathbf{u}_{d}$ based on the rotational invariance property as in (8).

8) Extract the estimated angle value of $\phi$ from (9).

9) Determine the frequency estimator using phase angle value of $\phi$ into (10).

10) Calculate the estimated BPM value equal to $\hat{f} \cdot 60 / T$.

\subsection{Healthcare Platform for Real-Time Monitoring and Evaluation}

The software architecture of the proposed application is divided into five layers, as depicted in Figure 4.

In the Presentation Layer of Figure 4, a login screen for authentication and registration of new users is defined. This feature allows users to register and identify their medical credentials and securely store their information in the application servers. 


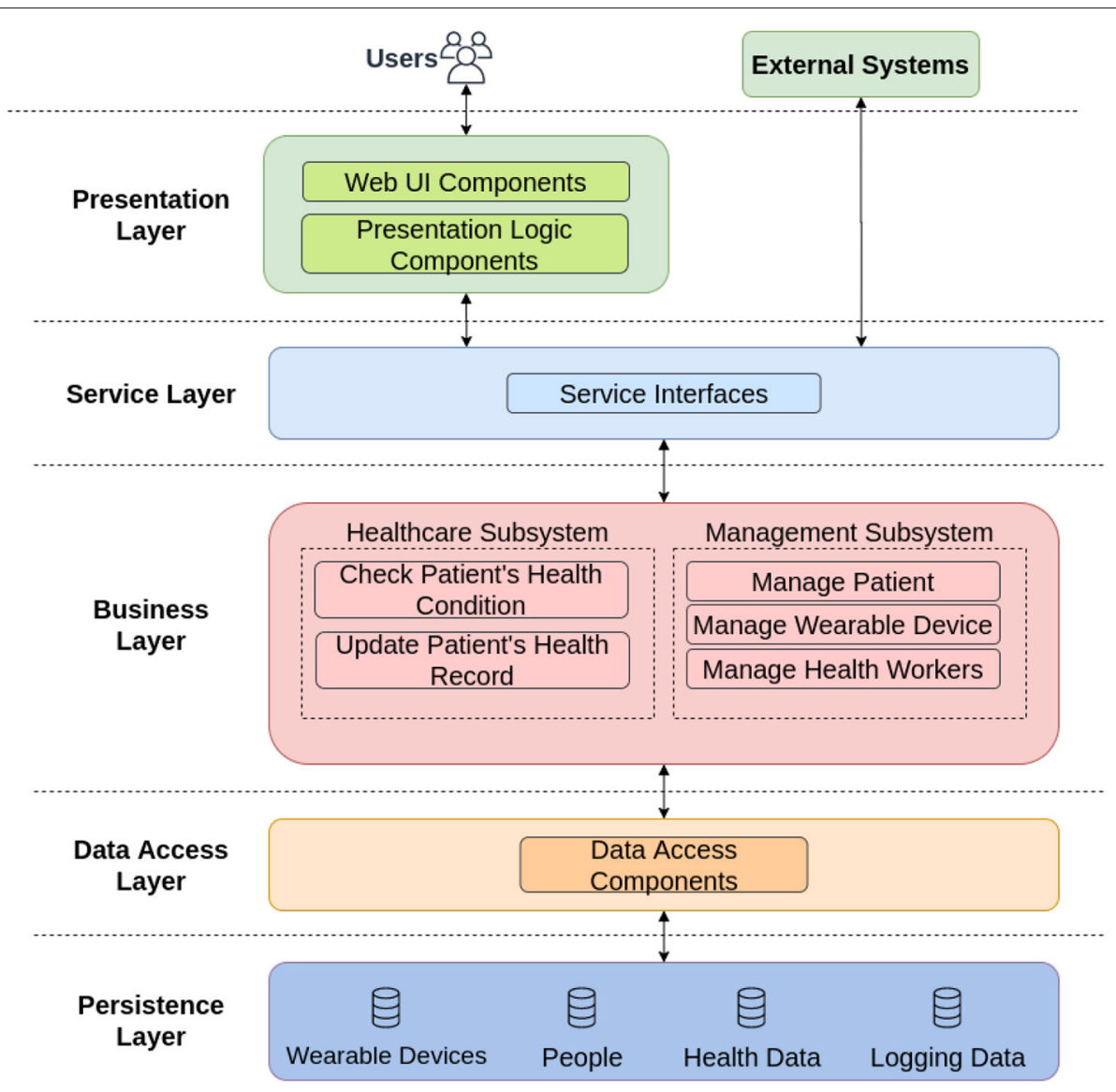

FIGURE 4 | Software architecture for the developed real-time monitoring healthcare application.

The Service Layer of Figure 4 exposes the business logic implemented in the software to potential consumers. One example of an external system is the concentrator, responsible for uploading data from wearable devices.

The Business Layer of Figure 4 is the logic behind the platform. We divided this layer into two blocks, in which the Healthcare Subsystem is used to check the data generated from the patient. The other block, called Management Subsystem, has an administrative function, tasked with supervising and managing multiple patients, wearable devices and healthcare workers.

The Data Access Layer of Figure 4 contains components to abstract the logic required to access the data stores. Such components provide common data access functionality, isolating the upper layers from the specific database technology, and making the application easier to maintain and configure.

The Persistence Layer of Figure 4 provides several advantages to the software since it is more efficient to save and retrieve data and provide for the whole application. In our context, we have four data sources, namely: Wearable Devices, People, Health Data, and Logging Data.

One advantage of the proposed online application in the solution framework is the interaction with the end-user. Using the platform, healthcare workers can track multiple users' health conditions in a centralized and reliable manner. Note that the proposed real-time monitoring platform can be integrated into other MWHD, as exemplified in Prettz et al. (2017), with the usage of a commercial MWHD.

Even though secure data communication is not a focus of this paper, it is essential to highlight that a distributed system such as this proposed healthcare application needs a fully distributed security system, such as proposed in Ferreira and de Sousa Jr. (2017). As it also comprises interactions according to the Internet of Things (IoT) paradigm and involves personal data, a particularly lightweight protocol for the authentication of devices, as proposed in de Almeida et al. (2018), is also paramount.

\section{EXPERIMENTAL VALIDATION}

To evaluate the performance of the proposed ESPRIT-based HR estimation approach in section 3.2, measurements using the PPG sensor equivalent to that of section 3.1 are considered. Unfortunately, the MAX30100 sensor used for this experimental validation from Maxim Integrated (2014) has an analog-to-digital 
converter (ADC) with lower resolution than that available in the MAX30102 in Maxim Integrated (2018) sensor present in the MWHD prototype, and also different possible parameter configurations. Nevertheless, we believe the results presented here are extensible to the proposed MWHD due to equivalences in both sensors' PPG technology.

The experimental validation trials were performed by one voluntary person that was asked to perform specific activity levels to produce different heart rate observations for the measured values. The voluntary person wore a medical-grade PPG devicefor ground truth-along with our proposed PPG measuring device. For each considered parameter, a total of five samples were captured, with a total duration of $10 \mathrm{~s}$ each. Initially, the voluntary was in rest, and then performed intense cardiovascular exercise for a defined time interval. Then, the voluntary rested after the cardiovascular exercise.

Initially, signal pre-processing is performed on the sampled data to eliminate artifacts and other detrimental factors that hinder each HR estimator's performance. Then, such processes are employed to account for signal processing present in PPG systems' real operation, maintaining this work's scope.

Motion artifacts are removed using an outlier detector that removes abruptly varying artifacts from the signal based on the derivative's high absolute values between consecutive samples. A low-frequency blocker filter-as proposed in Smith (2008)—is implemented with $R=0.95$ to filter most of the low frequency noise in the signal. A 6-th order Butterworth low-pass filter with a cutoff frequency of $4 \mathrm{~Hz}$ is applied to attenuate high-frequency noise. It leaves an effective bandwidth that can detect an HR of up to 240 BPM.

To establish a referential target value for the real HR value, we employ a medical oximeter model ELERA SH-K3 that measures HR and pulse oximetry using red and infrared light for transmissive PPG, worn on the user's finger. Immediately before and after each measurement, the HR values of the device were logged. This gives an initial and final reference value for the oximeter in the measurement time frame, allowing for $\mathrm{HR}$ variation during the experiment to be taken into account. The values measured after the experiment are then used as ground truth values for calculating the RMSE of each estimator. As a result, we believe that the sensors' final readings better reflect the BPM values during the experiment, as they include the same time window of the sample measurements.

After processing the samples with the procedures described, the same PPG sample data points were input in each of the compared algorithms, generating the estimated heart frequency for each sample. These estimated values were then compared to the ground truth values for each sample. Results are expressed in terms of the RMSE, given by:

$$
\operatorname{RMSE}(f, \hat{f})=\sqrt{\frac{1}{n_{\text {samples }}} \sum_{i=0}^{n_{\text {samples }}-1}\left(f_{i}-\hat{f}_{i}\right)^{2}}
$$

Calculated for the estimator $\hat{f}_{i}$, referenced either from the oximeter's ground truth value $f_{i}$ as read at the end of each measurement. After assessing each sample's individual RMSE, the general RMSE value for each algorithm was generated in each parameter's category. The performance comparison among the algorithms was based on the resulting general RMSE value for each algorithm in the categories considered.

The algorithms' scripts are developed in MATLAB version 2018a and were tested on Windows. Licensing is required for non-academic use of the software. The code used in this experimental validation is available on GitHub in Pinheiro (2021). Samples used are available on request.

For experimental validation, the PPG sensor configurations vary to evaluate each algorithm's capabilities and robustness. Initially, the IR LED current level, which controls its transmission power, is investigated using the values made available on the sensor. Lower IR current levels decrease the device's energy consumption. However, as the LED power also decreases, the detected signal is weaker, which may increase the estimation error. Configurations from 30.6 to $50 \mathrm{~mA}$ saturated the sensor's ADC, generating no meaningful data. The remaining measurements were processed with a signal time window of $5 \mathrm{~s}$ and a sample rate of $100 \mathrm{~Hz}$, amounting to 500 samples. In Figure 5, results are presented considering the oximeter reference, read right after experimental measurements ended.

According to Figure 5, the proposed algorithm based on ESPRIT resulted in lower RMSE values for most of the current values considered. Moreover, the proposed algorithm could perform more accurately at lower current levels, indicating saving more power.

In Figure 6, we varied the IR LED pulse width. Longer pulse widths increase power consumption and the detected signal's length, enabling the $\mathrm{ADC}$ to settle in a more precise value. Consequently, the ADC resolution available at the sensor is dependent on the selected pulse width, whose possible values are shown in Table 1. Experimental data was gathered, fixing the LED current level at $27.1 \mathrm{~mA}$ and sample rate at $100 \mathrm{~Hz}$. Data were processed with 500 samples, corresponding to a $5 \mathrm{~s}$ time window. The RMSE of the estimations is presented by the final readings of the oximeter reference considered in Figure 6.

The estimator results are consistent with the previous test case, with the proposed algorithm generally having lower RMSE values. These tests showed significant results regarding algorithm performance with reduced pulse width and ADC resolution. Pulse width shorter than $50 \%$ of the maximum value is shown to degrade estimation precision considerably. In addition, the shortest pulse width generated higher error values than other configurations. This may be due to signal ripple caused by pulsewidth modulation (PWM) operation, which is more substantial the pulse is shorter. Another factor to consider is the lower ADC resolution corresponding to lower LED pulse width. Therefore, a lower sampling resolution can further impair the output PPG parameter estimation along with suspected ripple noise.

We also validate variations in the sample rate to corroborate with the previous experimental stages. A higher sampling rate decreases signal aliasing, yet the more massive samples per second require the $\mathrm{ADC}$ to settle in a lower resolution. The experimental results obtained show that higher sample rates have decreased precision in HR, possibly due to the imposed reduction in $\mathrm{ADC}$ resolution in each configuration tested, as can be seen in Table 2 . Hence, a sample rate of $100 \mathrm{~Hz}$ is considered 

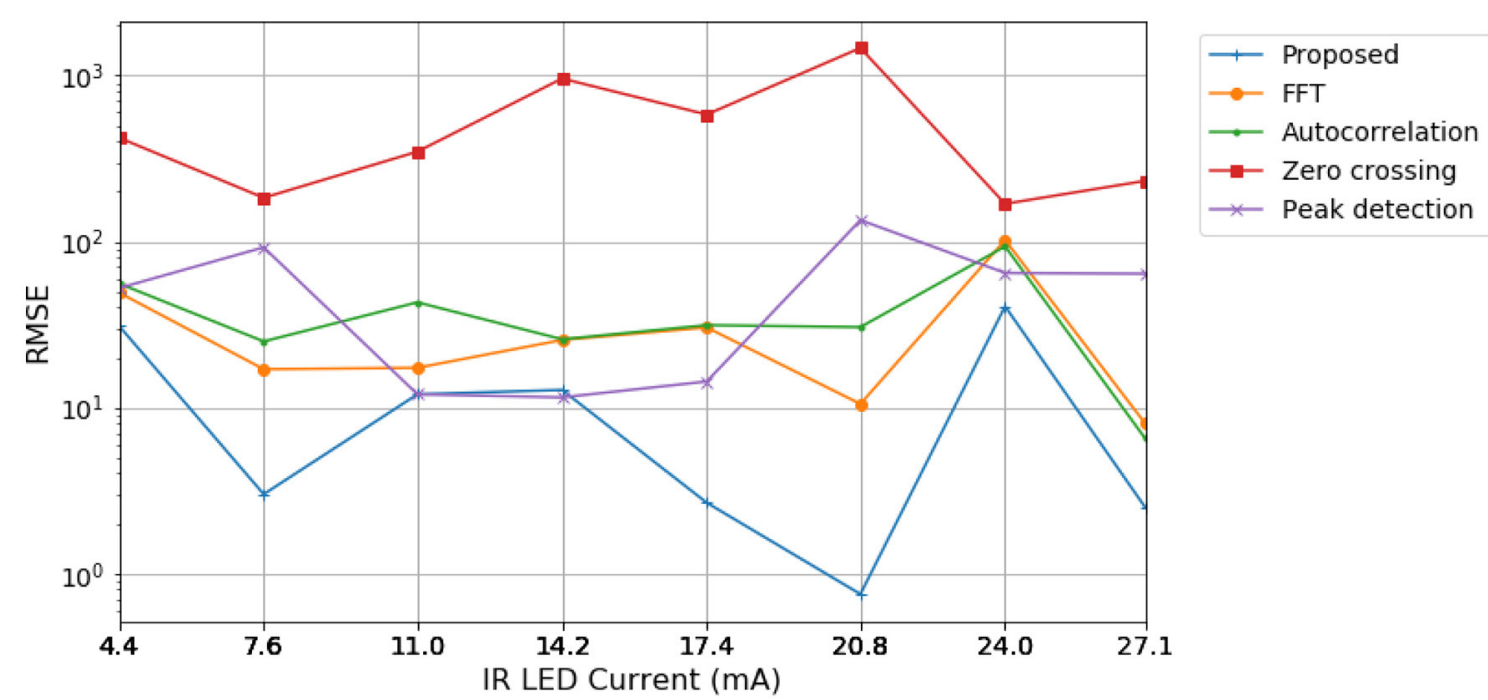

FIGURE 5 | RMSE of estimated BPM values for each algorithm for different current level configurations, referenced to values read by the oximeter.
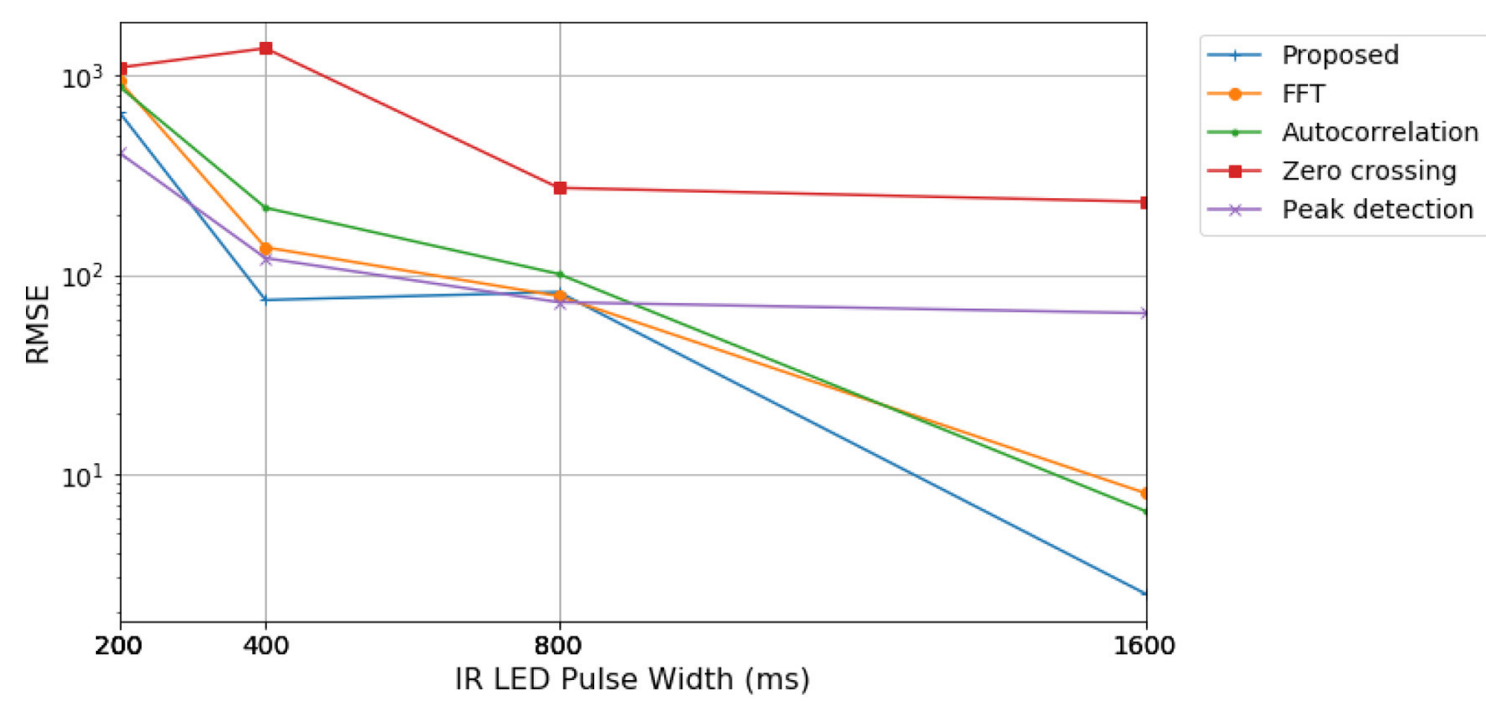

FIGURE 6 | RMSE of estimated BPM values for different pulse width and corresponding ADC resolution configurations, referenced to values read by the oximeter.

TABLE 1 | LED pulse width configurations tested and the correspondent ADC resolution.

\section{Pulse width $(\mu \mathrm{s})$}

ADC resolution (bits)

\begin{tabular}{ll}
\hline 1,600 & 16 \\
800 & 15 \\
400 & 14 \\
200 & 13
\end{tabular}

advantageous since it maintains a larger signal bandwidth and maximum ADC resolution.

The proposed algorithm based on ESPRIT showed consistent results throughout the experiments performed. Moreover, it
TABLE 2 | Maximum available ADC resolution for each sample rate configuration tested.

\begin{tabular}{lr} 
Sample rate $\mathbf{( H z )}$ & ADC resoluti \\
\hline 50 & 16 \\
100 & 16 \\
167 & 15 \\
200 & 15 \\
400 & 14
\end{tabular}

generally has lower RMSE values for lower IR LED current configurations and lower sampling frequencies, following the general trend in the pulse width variation. Hence, the increased 
TABLE 3 | Access times to different test requests performed to the API of the real-time monitoring application.

\begin{tabular}{lc}
\hline Action & Access time (ms) \\
\hline Retrieve data from feed page & 43.87 \\
Access patient's profile & 95.0 \\
Add new patient & 97.70 \\
List teams & 61.19 \\
Request patients list & 213.08 \\
Add new healthcare worker & 36.01
\end{tabular}

precision demonstrates the potential for power-saving and more resilient performance in challenging scenarios.

An analysis of power consumption of the proposed MWHD was carried out in de Assis (2018) and is summarized here for further experimental validation. As a result, the current consumption was measured as $2.032 \mathrm{~mA}$ on average. That leads to a total battery autonomy of the MWHD of approximately $73 \mathrm{~h}$, about a small-sized $150 \mathrm{mAh}$ LiPo battery. Moreover, that enables the MWHD to be used for around 3 days without recharging, adding comfort to senior patients' use.

The connection between the MWHD and the real-time monitoring application is possible due to the Representational State Transfer (REST) protocol. We built an Application Programming Interface (API) to perform this connection with the database based on this protocol. We computed the times in different requests for the different pages of the platform. The results are presented in Table 3 .

As shown in Table 3, the low latency of access validates that the proposed online platform can be used for realtime monitoring.

\section{CONCLUSION}

This paper proposes a multi-sensor wearable health device framework and a real-time monitoring application with highresolution parameter estimation. A complete solution enables the needs of elderly patients to be better accommodated whilst ensuring that the latest COVID-19 related health protocols are observed. The proposed hardware includes step counting, pulse oximetry, HR, and temperature sensors. In addition, the proposed MWHD optimizes battery usage by using BLE. In terms of parameter estimation, the embedded system programmed with high-resolution algorithms processes signals from the multiple sensors used, allowing an improved estimation of the steps and HR. Finally, the patient's medical information is reliably provided to the healthcare workers by the real-time monitoring application.

Future works include a possible hardware modification to include an additional processor dedicated to data measurement and processing, ensuring that the proposed high-resolution ESPRIT algorithm runs alongside the BLE interface while conserving the most battery power. Furthermore, the proposed health application is considered to evolve with parallel and distributed processing based on microservices over GPU grids, thus allowing computing strategies to process multiple dataflows from devices for the sake of machine learning and pattern recognition. As mentioned, it will also leverage the exploitation of federated learning techniques over dataflows from multiple MWHD, thus allowing monitoring and artificial reasoning on grouped data from patients under supervision.

\section{DATA AVAILABILITY STATEMENT}

The raw data supporting the conclusions of this article will be made available by the authors, without undue reservation.

\section{ETHICS STATEMENT}

Ethical review and approval was not required for the study on human participants in accordance with the local legislation and institutional requirements. The patients/participants provided their written informed consent to participate in this study.

\section{AUTHOR CONTRIBUTIONS}

GP and BP: formal analysis, investigation, software, and writing - original draft. RM and GS: validation, investigation, and supervision. FM: resources. FM and EJ: visualization. RdS and FM: funding acquisition. EJ: validation and data curation. JdC and RdS: conceptualization and supervision. JdC: formal analysis, methodology, and writing - original draft. RdS: writing - review and editing. All authors have read and agreed to the published version of the manuscript.

\section{FUNDING}

This work is supported in part by $\mathrm{CNPq}$ - Brazilian National Research Council (No. PQ-2 312180/2019-5 on Cybersecurity No. 465741/2014-2), in part by the National Auditing Department of the Brazilian Health System SUS (No. DENASUS 23106.118410/2020-85), in part by the Brazilian Ministry of the Economy (Nos. DIPLA 005/2016 and ENAP 083/2016), in part by the Administrative Council for Economic Defense (No. CADE 08700.000047/2019-14), in part by the General Attorney of the Union (No. AGU 697.935/2019), and in part by the General Attorney's Office for the National Treasure (No. PGFN 23106.148934/2019-67).

\section{ACKNOWLEDGMENTS}

The authors would like to acknowledge the support of $\mathrm{CNPq}$ - Brazilian National Research Council, CAPES - Brazilian Higher Education Personnel Improvement Coordination, FAPDF - Brazilian Federal District Research Support Foundation, DENASUS - National Auditing Department of the Brazilian Health System SUS, AGU - General Attorney of the Union, ABIN - Brazilian Intelligence Agency, ME - Brazilian Ministry of the Economy, CADE - Administrative Council for Economic Defense, and PGFN - General Attorney's Office for the National Treasure. The authors would also like to thank Prof. José Alfredo Ruiz Vargas for supporting us as a co-author of this paper. Icons in Figure 1 were produced with Freepik from www.flaticon.com. 


\section{REFERENCES}

Allen, J. (2007). Photoplethysmography and its application in clinical physiological measurement. Physiol. Measure. 28:R1. doi: 10.1088/0967-3334/28/3/R01

Alwashmi, M. F. (2020). The use of digital health in the detection and management of covid-19. Int. J. Environ. Res. Public Health 17:2906. doi: 10.3390/ijerph17082906

Buccelletti, E., Gilardi, E., Scaini, E., Galiuto, L., Persiani, R., Biondi, A., et al. (2009). Heart rate variability and myocardial infarction: systematic literature review and metanalysis. Eur. Rev. Med. Pharmacol. Sci. 13, 299-307. Available online at: https://www.europeanreview.org/article/650

Chung, H., Ko, H., Lee, H., and Lee, J. (2020). Deep learning for heart rate estimation from reflectance photoplethysmography with acceleration power spectrum and acceleration intensity. IEEE Access 8, 63390-63402. doi: 10.1109/ACCESS.2020.2981956

Coffen, B., Scott, P., and Mahmud, M. S. (2020). "Real-time wireless health monitoring: an ultra-low power biosensor ring for heart disease monitoring," in 2020 International Conference on Computing, Networking and Communications (ICNC) (Big Island, HI: IEEE), 626-630. doi: 10.1109/ICNC47757.2020.9049814

Costantino, G., Solbiati, M., Elli, S., Paganuzzi, M., Massabó, D., Montano, N., et al. (2021). Utility of hospitalization for elderly individuals affected by COVID-19. PLOS ONE 16:e250730. doi: 10.1371/journal.pone.0250730

de Almeida, M., de Sousa, R. T. Jr., Garcia Villalba, L. J., and Kim, T.-H. (2018). New dos defense method based on strong designated verifier signatures. Sensors 18:2813. doi: 10.3390/s18092813

de Assis, M. F. P. (2018). Implementacao de Algoritmos em Sistemas Embarcados de Baixo Consumo Dotados de Radio Sem Fio e Analise de Perfil de Consumo Energatico. Trabalho de Conclusao de Curso, Universidade de Brasalia, Faculdade de Tecnologia, Departamento de Engenharia Elatrica, Brasalia.

de Freitas, E. P., da Costa, J. P. C. L., de Almeida, A. L. F., and Marinho, M. (2012). "Applying mimo techniques to minimize energy consumption for long distances communications in wireless sensor networks," in Internet of Things, Smart Spaces, and Next Generation Networking, eds S. Andreev, S. Balandin, and Y. Koucheryavy (Berlin; Heidelberg: Springer), 379-390. doi: 10.1007/978-3-642-32686-8_35

Durán-Vega, L. A., Santana-Mancilla, P. C., Buenrostro-Mariscal, R., ContrerasCastillo, J., Anido-Rifón, L. E., Garcia-Ruiz, M. A., et al. (2019). An IoT system for remote health monitoring in elderly adults through a wearable device and mobile application. Geriatrics 4:34. doi: 10.3390/geriatrics4020034

Ferreira, H. G. C., and de Sousa, J.r., R. T. (2017). Security analysis of a proposed internet of things middleware. Cluster Comput. 20, 651-660. doi: $10.1007 / \mathrm{s} 10586-017-0729-3$

Godfrey, A. (2017). Wearables for independent living in older adults: gait and falls. Maturitas 100, 16-26. doi: 10.1016/j.maturitas.2017.03.317

Grand View Research (2020). Wearable Medical Device Market Size, Share Trends Analysis Report By Product Type (Diagnostic, Therapeutic, Respiratory), By Site (Strap/Clip/Bracelet, Handheld), By Application, And Segment Forecasts, 2020 2027. Technical report, Grand View Research.

Kekade, S., Hseieh, C.-H., Islam, M. M., Atique, S., Khalfan, A. M., Li, Y.C., et al. (2018). The usefulness and actual use of wearable devices among the elderly population. Comput. Methods Programs Biomed. 153, 137-159. doi: 10.1016/j.cmpb.2017.10.008

Kolasinska, A., Quadrio, G., Gaggi, O., and Palazzi, C. E. (2018). “Technology and aging," in Proceedings of the 4th EAI International Conference on Smart Objects and Technologies for Social Good - Goodtechs'18 (Bologna: ACM Press). doi: $10.1145 / 3284869.3284884$

Lampoltshammer, T. J., Pignaton de Freitas, E., Nowotny, T., Plank, S., Da Costa, J. P. C. L., Larsson, T., et al. (2014). Use of local intelligence to reduce energy consumption of wireless sensor nodes in elderly health monitoring systems. Sensors 14, 4932-4947. doi: 10.3390/s140304932

Manolakis, D. G., Ingle, V. K., and Kogon, M. S. (2005). Statistical and Adaptive Signal Processing. Artech House, Inc.

Marinho, M. A. M., de Freitas, E. P., Lustosa da Costa, C. J. P., and de Almeida, F. A. L. (2013). "Using cooperative mimo techniques and uav relay networks to support connectivity in sparse wireless sensor networks," in 2013 International Conference on Computing, Management, and Telecommunications (Ho Chi Minh City: ComManTel), 49-54. doi: 10.1109/ComManTel.2013.6482364
Maxim Integrated (2014). MAX30100 Pulse Oximeter and Heart-Rate Sensor IC for Wearable Health. Maxim Integrated. Rev. 0.

Maxim Integrated (2016). MAX30205 Human Body Temperature Sensor. Maxim Integrated. Rev. 0.

Maxim Integrated (2018). MAX30102 High-Sensitivity Pulse Oximeter and HeartRate Sensor for Wearable Health. Maxim Integrated. Rev. 1.

Panwar, M., Gautam, A., Biswas, D., and Acharyya, A. (2020). PP-Net: a deep learning framework for PPG based blood pressure and heart rate estimation. IEEE Sensors J. 10000-10011. doi: 10.1109/JSEN.2020.2990864

Phua, J., Weng, L., Ling, L., Egi, M., Lim, C.-M., Divatia, J. V., et al. (2020). Intensive care management of coronavirus disease 2019 (covid19): challenges and recommendations. Lancet Respirat. Med. 8, 506-517. doi: 10.1016/S2213-2600(20)30161-2

Pinheiro, G. (2021). gabrielpmp/esprit-hr: Release version. (Brasília: Gabriel Passos Moreira Pinheiro).

Prettz, J. B., da Costa, J. P. C. L., Alvim, J. R., Miranda, R. K., and Zanatta, M. R. (2017). "Efficient and low cost MIMO communication architecture for smartbands applied to postoperative patient care," in 2017 Second Russia and Pacific Conference on Computer Technology and Applications (RPC) (Vladivostok: IEEE), 1-5. doi: 10.1109/RPC.2017.8168055

Proakis, J. G. (2013). Digital Signal Processing: Pearson New International Edition. Pearson Group.

Przybyło, J. (2019). Continuous distant measurement of the user's heart rate in human-computer interaction applications. Sensors 19:4205. doi: $10.3390 / \mathrm{s} 19194205$

Puranik, S., and Morales, A. W. (2020). Heart rate estimation of PPG signals with simultaneous accelerometry using adaptive neural network filtering. IEEE Trans. Cons. Electron. 66, 69-76. doi: 10.1109/TCE.2019.2961263

Rega, D. G., Miranda, R. K., Javidi, E., Maranhao, J. P. A., da Costa, J. P. C. L., and Pinheiro, G. P. M. (2019). "ESPRIT-based step count for wearable devices," in 2019 13th International Conference on Signal Processing and Communication Systems (ICSPCS) (Gold Coast, QLD: IEEE), 1-5. doi: 10.1109/ICSPCS47537.2019.9008702

Reis, P. M. G. I., da Costa, J. P. C. L., Miranda, R. K., and del Gado, G. (2016). "Audio authentication using the kurtosis of esprit based enf estimates," in 2016 10th International Conference on Signal Processing and Communication Systems (Surfers Paradise, QLD: ICSPCS), 1-6. doi: 10.1109/ICSPCS.2016.7843308

Reis, P. M. G. I., da Costa, J. P. C. L., Miranda, R. K., and Del Galdo, G. (2017). Esprit-hilbert-based audio tampering detection with SVM classifier for forensic analysis via electrical network frequency. IEEE Trans. Inform. Forensics Secur. 12, 853-864. doi: 10.1109/TIFS.2016.2636095

Rezende, L. F. M., Thome, B., Schveitzer, M. C., de Souza-Júnior, P. R. B., and Szwarcwald, C. L. (2020). Adults at high-risk of severe coronavirus disease-2019 (covid-19) in Brazil. Revista de Saúde Pública 54:50. doi: 10.11606/s1518-8787.2020054002596

Roy, R., and Kailath, T. (1989). Esprit-estimation of signal parameters via rotational invariance techniques. IEEE Trans. Acoust. Speech Signal Process. 37, 984-995. doi: 10.1109/29.32276

Santamaria, I., Pantaleon, C., and Ibanez, J. (2000). A comparative study of highaccuracy frequency estimation methods. Mech. Syst. Signal Process. 14, 819-834. doi: 10.1006/mssp.2000.1321

Scholkmann, F., Boss, J., and Wolf, M. (2012). An efficient algorithm for automatic peak detection in noisy periodic and quasi-periodic signals. Algorithms 5, 588-603. doi: 10.3390/a5040588

Smith, J. (2008). Introduction to Digital Filters: With Audio Applications.W3K.Texas Instruments (2016). CC2640 Simple Link TM Bluetooth R Wireless. MCU.

Texas Instruments (2016). CC2640 SimpleLink ${ }^{T M}$ Bluetooth $^{\circledR}$ Wireless MCU. Texas Instruments.

Wang, M., Li, Z., Zhang, Q., and Wang, G. (2019). Removal of motion artifacts in photoplethysmograph sensors during intensive exercise for accurate heart rate calculation based on frequency estimation and notch filtering. Sensors 19:3312. doi: 10.3390/s191 53312

Wu, T., Wu, F., Qiu, C., Redoute, J.-M., and Yuce, M. R. (2020). A rigidflex wearable health monitoring sensor patch for IoT-connected healthcare applications. IEEE Internet Things J. 6932-6945. doi: 10.1109/JIOT.2020. 2977164 
Xiong, J., Cai, L., Wang, F., and He, X. (2017). SVM-based spectral analysis for heart rate from multi-channel wppg sensor signals. Sensors 17:506. doi: $10.3390 /$ s17030506

Zhang, F., Yeddanapudi, M., and Mosterman, P. J. (2008). Zero-crossing location and detection algorithms for hybrid system simulation. IFAC Proc. 41, 7967-7972. doi: 10.3182/20080706-5-KR-1001.01346

Conflict of Interest: The authors declare that the research was conducted in the absence of any commercial or financial relationships that could be construed as a potential conflictof interest.

Publisher's Note: All claims expressed in this article are solely those of the authors and do not necessarily represent those of their affiliated organizations, or those of the publisher, the editors and the reviewers. Any product that may be evaluated in this article, or claim that may be made by its manufacturer, is not guaranteed or endorsed by the publisher.

Copyright (ㄷ) 2022 Pinheiro, Miranda, Praciano, Santos, Mendonça, Javidi, da Costa and de Sousa. This is an open-access article distributed under the terms of the Creative Commons Attribution License (CC BY). The use, distribution or reproduction in other forums is permitted, provided the original author(s) and the copyright owner(s) are credited and that the original publication in this journal is cited, in accordance with accepted academic practice. No use, distribution or reproduction is permitted which does not comply with these terms. 